\title{
A further study of melodic channeling
}

\author{
DAVID BUTLER \\ School of Music, Ohio State University, Columbus, Ohio 43210
}

\begin{abstract}
Subjects listened to repetitive presentations of $\mathrm{C}$ major scale patterns and simple two-part contrapuntal specimens, both dichotically and in a stereophonic free sound field. All scalar and melodic patterns were presented so that successive tones alternated from ear to ear: when a component of one scale or melody was routed through one speaker or headphone, the concurrent member of the other scale or melodic pattern was routed through the other speaker or headphone, and vice versa. The stimulus parameters of spectral contour and envelope characteristics, duration, melodic pattern, and loudness were varied, and a testing procedure was designed to minimize any bias in responses which might be produced by learning, immediate memory, or vocal limitations of subjects. Virtually all responses $(95.2 \%$ to scalar stimuli, $99.1 \%$ to melodic stimuli) indicated that the subjects channeled stimuli by pitch range, and not by ear of input. When spectral contours routed through the separate headphones or speakers were noticeably dissimilar, no subject perceived that this timbral inequality switched from ear to ear: all subjects heard an overall change in tone quality which pervaded both scalar or melodic sequences, and which apparently emanated from both headphones or speakers.
\end{abstract}

Recent studies (Deutsch, 1974, 1975, 1978; Deutsch \& Roll, 1976) indicate that when streams of pitch information are presented dichotically such that adjacent members of the streams are switched from ear to ear, various auditory illusions result. When C major scales were used as stimuli (Deutsch, 1975), it was found that subjects uniformly perceived pitch patterns which were ordered within higher and lower pitch bands, and were not ordered by ear of input. Figure la shows the pitch sequences routed to the separate ears; Deutsch found that all subjects perceived these sequences as four-note (tetrachordal) melodic patterns which musicians commonly call "arch contours" (Figure 1b). No subjects perceived these sequences as eight-note scale patterns in contrary motion, such as that shown in Figure 1c.

The present study investigates the extent to which certain experimental controls and procedures may be modified without undoing the perceptual phenomenon of channeling. In the studies cited above, stimuli consisted of sine tones generated by computercontrolled oscillators; these tones were presented at equal sound pressure levels (SPL) of $75 \mathrm{~dB}$. All tones had a duration of $250 \mathrm{msec}$. Stimuli were limited to presentations of octaves (Deutsch, 1974, 1978; Deutsch \& Roll, 1976) or of C major scale patterns (Deutsch, 1975).

When one attempts to judge the musical significance of these findings, several questions immediate-

This work was supported by an Ohio State University Small Research Grant. Requests for reprints should be sent to David Butler, School of Music, The Ohio State University, Columbus, Ohio 43210. I thank Dr. T. Wells, Director of the OSU Electronic Music Studio, for technical support. ly arise: (1) Although some newer works may have been written specifically for dichotic performance, most music is still heard binaurally, whether through stereo headphones or in a free sound field environment. How might binaural (i.e., stereophonic free sound field) presentation affect the channeling phenomenon? (2) Since very few musical tones, even those found in electronic music compositions, are unmodulated sine tones, do we segregate streams of complex tones into channels according to the pitch bands which relate to their fundamental frequencies? (3) Computer-controlled sound generation allows for a degree of perfection seldom, if ever, achieved by even outstanding performers. What effects might such imperfections as improperly timed attacks, inequal strengths of attack, inequal durations (both articulative and metrical), and the presence of anomalous transient information, all of which exist in abundance in musical performances, have on the channeling phenomenon? (4) It might be argued that octaves and major scales are such common, rudimen-

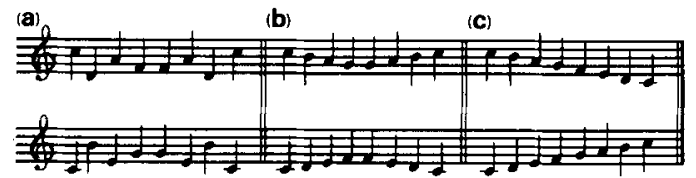

Figure 1. (a) Representation of the pitch sequence employed by Deutsch (1975) and in Experiments 1 and 2 of this study. (b) Representation of the opposed tetrachordal pattern most often reported (95.2\% of responses) by subjects in Experiments 1 and 2. (c) Representation of the opposed eight-note scalar pattern reported in $4.8 \%$ of the responses in Experiments 1 and 2, all given by subjects working in a stereophonic free sound field environment. 
tary constructs in western music that channeling, given the nature of these stimuli, is a demonstration of learning-that is, demonstration of a cultural bias. Do we channel more-complex melodies with which we can assume a limited amount of familiarity? (5) Finally, it might also be argued that testing procedures might greatly influence experimental results: In the studies cited above, subjects were instructed either to give a verbal report on their perceptions, or to "shadow sing" along with the stimuli. Could experimental results have been affected by idiosyncracies of memory or through vocal limitations of subjects?

In the present study, certain experimental controls and procedures were modified, and additional stimuli were generated, in an attempt to seek adequate responses to these questions.

\section{METHOD}

\section{Stimuli}

Experiment 1. The pitch sequence employed in this experiment is shown in Figure 1a. A C major scale pattern was presented both in ascending and descending form, such that when a component of the ascending scale was routed through the left headphone or speaker, a component of the descending scale was routed through the right, and vice versa. Each sequence was presented repetitively eight times without pause. All tones were approximately $250 \mathrm{msec}$ in duration (M. M. quarter note $=240$ ), with no gaps between adjacent tones. During the experiment, each subject heard five such repetitive sequences. The first two were identical, with pitch series composed of sine tones. The third sequence consisted of the same pitch series, but played on a piano. The fourth series was a repetition of the third, with the left-headphone or speaker signal modified so that all ascending spectral ingredients from $800 \mathrm{~Hz}$ were amplified $5 \mathrm{~dB}$. The fifth sequence was another modification of the third, with all ascending spectral ingredients from $800 \mathrm{~Hz}$ amplified $10 \mathrm{~dB}$, in the left-headphone or speaker signal only.

Experiment 2. The purpose in this experiment was to check for possible order effects in Experiment 1. The fifth and first sequences from Experiment 1 were presented, in that order. Other than this order change, the stimuli were unaltered.

Experiment 3. The two-part contrapuntal patterns used in this experiment are shown in Figures $2 a$ and $2 c$. As in the other experiments, successive tones in the pairs of melodic patterns were alternated from ear to ear, resulting in the disjunct patterns

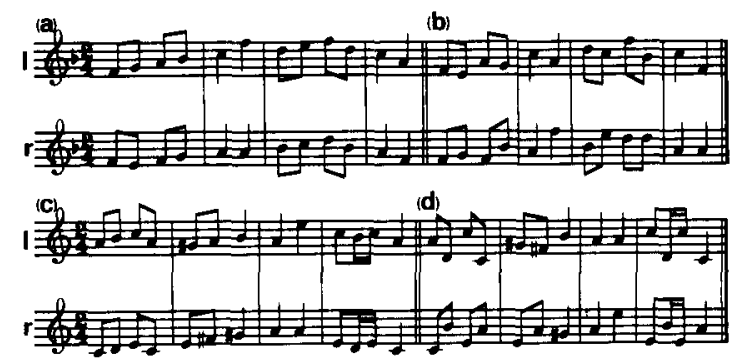

Figure 2. (a and c) Representation of the contrapuntal specimens as they were notated by subjects in Experiment 3. (b and d) Representation of the switched melodic patterns routed to the left and right headphones or speakers.
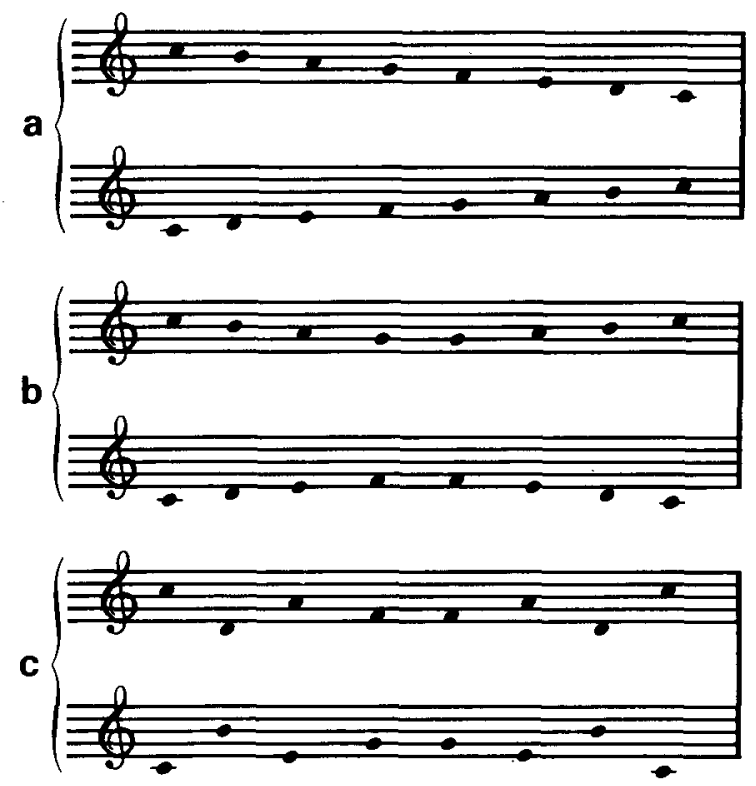

Figure 3. Representation of the visual cue-sheet given subjects in Experiment 1.

shown in Figures $2 \mathrm{~b}$ and $2 \mathrm{~d}$. Separate recordings of these stimuli were also made, such that the frequency responses on the two tracks were inequal. The first modification consisted of increasing the intensity of all spectral components from 250 to $630 \mathrm{~Hz}$ by $10 \mathrm{~dB}$ in the left-headphone or speaker signal only, while the response curve of the other signal was left flat. The second modification consisted of increasing the intensity of all ascending spectral ingredients from $800 \mathrm{~Hz}$ by $10 \mathrm{~dB}$, again in the leftheadphone or speaker signal only. These patterns were played on a piano at a moderate tempo (M.M. quarter note $=116)$.

The tones for all three experiments were taken from an equaltempered scale $(A=440 \mathrm{~Hz})$. Although sound pressure levels at the headphones or speakers were always kept equal, subjects were allowed to adjust the master loudness control to a level that they considered comfortable. These preferred levels of loudness fell within the SPL range of about 45 to $75 \mathrm{~dB}$.

\section{Procedure}

Each subject was individually tested. He was instructed to take melodic dictation from the stimulus tapes, and was allowed to listen to the stimuli as often as he desired. In each experiment, one test group took melodic dictation through headphones (dichotic presentation), and a second group followed this procedure in a stereophonic free sound field (binaural presentation) environment. The subjects who worked in the free sound field environment were seated at a point equidistant from the two speakers.

Experiment 1. Subjects notated the five examples and were asked to indicate, in margin notes, any disparities that they heard among the examples. The subjects were told that these disparities might include differences in loudness, that one example might be presented in a higher or lower octave register than another, or that one example might differ from another in tone quality. After this phase of the test was completed, the tape was rewound and the subjects were asked to indicate the apparent source of each scale pattern. Finally, after the tape was again rewound, the subjects were given a sheet containing the examples shown in Figure 3, and were asked to indicate which of the three notated examples best represented what they were about to hear. The scale patterns were then presented a final time. 
Experiment 2. Subjects notated the two examples, and were instructed to note any disparities that they heard between these examples. As in Experiment 1, the subjects were told that these disparities might include differences of loudness, that the second example might be presented an octave above or below the first, or that the first example might differ from the second in tone quality.

Experiment 3. Subjects notated the two examples (Figures $2 \mathrm{~b}$ and $2 \mathrm{~d}$ ). When the subjects were satisfied that they had properly notated the examples, they were allowed to hear the modified recordings of those examples, in which the spectral contour on one of the two tracks had been altered. At this point, the subjects were instructed, first, to indicate any changes of loudness, octave register, or tone quality that they perceived; and, second, to revise their notated copies of the examples to show any changes of pitch or duration that had become apparent. The tape was then rewound, and the subjects, as they listened to the original examples, were asked to indicate the apparent directional source of each melodic pattern (i.e., left and right headphone or speaker).

\section{Subjects}

The subjects were 66 students and faculty in the School of Music at The Ohio State University. Every subject possessed at least 1 year of training in music dictation as part of the music major curriculum, and had accumulated grades of "very good" or "excellent" at that skill. The subjects were paid for their services.

\section{Apparatus}

Tones were generated either by keyboard-controlled Moog 900 series sine-wave generators or by a piano. They were recorded at equal amplitude on tape, and were played to the subjects on a TEAC 2340SX tape recorder, either through Koss SP/3XC headphones or Altec A7-500-8 speakers. Spectral alterations were attained with a UREI 527-A monaural 27-band graphic equalizer.

\section{RESULTS}

\section{Experiment 1}

The stimuli were presented dichotically to six subjects, and binaurally to 10 subjects. Table 1 shows the responses of these subject groups in percentages.

Table 1

Results of Experiments 1 and 2 Expressed as Percentages of Responses

\begin{tabular}{lcc}
\hline & \multicolumn{2}{c}{ Presentation } \\
\cline { 2 - 3 } Reported Percept & \multicolumn{2}{c}{$\begin{array}{c}\text { Free } \\
\text { Sound }\end{array}$} \\
& Dichotic & Field \\
\hline & Experiment 1 \\
(a) Opposed Tetrachordal Arches & 100 & 90 \\
(b) Opposed Eight-Note Scales & 0.0 & 10 \\
(c) Disjunct Pitch Streams & 0.0 & 0.0 \\
& Experiment 2 \\
(a) Opposed Tetrachordal Arches & 100 & 95.8 \\
(b) Opposed Eight-Note Scales & 0.0 & 4.2 \\
(c) Disjunct Pitch Streams & 0.0 & 0.0 \\
\hline
\end{tabular}

Note-(a) The subject heard an opposed tetrachordal arch pattern as shown in Figure 1b; (b) the subject heard an opposed eight-note scale pattern as shown in Figure 1c; (c) the subject heard disjunct pitch streams as shown in Figure la.
Table 2

Responses to the Dichotic Presentation of Five Examples When Subjects Were Given the Visual Cues Shown in Figure 3

\begin{tabular}{cccccc}
\hline & \multicolumn{5}{c}{ Example } \\
\cline { 2 - 6 } Subject & 1 & 2 & 3 & 4 & 5 \\
\hline 1 & b & c & c & c & c \\
2 & c & b & c & c & b \\
3 & c & c & c & c & c \\
4 & b & b & b & b & b \\
5 & b & b & b & c & a \\
6 & b & b & b & b & b \\
\hline
\end{tabular}

Note-(a) The subject heard opposed eight-note scale patterns; (b) the subject heard opposed tetrachord arch patterns; (c) the subject heard a pair of disjunct pitch streams.

No subject perceived the disjunct melodic patterns shown in Figure 1a. Virtually all subjects perceived these pitch sequences as a pair of tetrachordal arches in contrary motion, as shown in Figure $1 b$. The only exceptional responses occurred within the free-soundfield subject group, two of whom notated eightnote scale patterns in contrary motion, as shown in Figure 1c. Interestingly, one of these subjects notated the eight-note scale patterns in response to Examples 1 and 2, but notated tetrachordal arch patterns in response to Examples 3, 4, and 5. The second subject notated tetrachordal arch patterns in response to Examples 1, 2, and 3, but notated eight-note scale patterns in response to Examples 4 and 5. Since 16 subjects collectively heard 80 examples, $93.8 \%$ of the responses indicated the perception of opposed tetrachordal arches, $6.2 \%$ of the responses indicated the perception of opposed eight-note scale patterns, and none of the responses indicated the perception of the disjunct stimulus sequence.

When the subjects were asked to listen to the examples once again, and to indicate which prenotated patterns (Figure 3 ) best represented their aural percepts, the responses became more varied.

Table 2 shows that a bare majority $(53 \%)$ of the responses from the dichotic subject group indicated the persistent perception of the opposed tetrachordal arch pattern, even when the subjects were aided by visual cues, and were told of the possible existence of the disjunct pattern shown in Figure 3c. The responses to Example 3, compared to responses to Examples 1 and 2, indicate that there is no remarkable difference in the subjects' responses to series of piano tones in contrast to series of sinusoids.

Table 3 shows that a strong majority (76\%) of the responses from the binaural subject population indicate the continued perception of the opposed tetrachordal arch patterns. Again, the responses to Example 3, which contained a series of unaltered piano tones, did not differ greatly from the responses to Examples 1 and 2 , which contained series of sine tones. 
Table 3

Responses to the Binaural Presentation of Five Examples When Subjects Were Given the Visual Cues Shown in Figure 3

\begin{tabular}{cccccc}
\hline & \multicolumn{5}{c}{ Example } \\
\cline { 2 - 6 } Subject & l & 2 & 3 & 4 & 5 \\
\hline 1 & $\mathrm{~b}$ & $\mathrm{~b}$ & $\mathrm{~b}$ & $\mathrm{~b}$ & $\mathrm{~b}$ \\
2 & $\mathrm{a}$ & $\mathrm{b}$ & $\mathrm{a}$ & $\mathrm{c}$ & $\mathrm{b}$ \\
3 & $\mathrm{a}$ & $\mathrm{a}$ & $\mathrm{c}$ & $\mathrm{c}$ & $\mathrm{b}$ \\
4 & $\mathrm{~b}$ & $\mathrm{~b}$ & $\mathrm{~b}$ & $\mathrm{~b}$ & $\mathrm{~b}$ \\
5 & $\mathrm{~b}$ & $\mathrm{~b}$ & $\mathrm{~b}$ & $\mathrm{c}$ & $\mathrm{c}$ \\
6 & $\mathrm{~b}$ & $\mathrm{~b}$ & $\mathrm{~b}$ & $\mathrm{~b}$ & $\mathrm{~b}$ \\
7 & $\mathrm{~b}$ & $\mathrm{~b}$ & $\mathrm{~b}$ & $\mathrm{~b}$ & $\mathrm{~b}$ \\
8 & $\mathrm{~b}$ & $\mathrm{~b}$ & $\mathrm{a}$ & $\mathrm{a}$ & $\mathrm{b}$ \\
9 & $\mathrm{~b}$ & $\mathrm{~b}$ & $\mathrm{~b}$ & $\mathrm{~b}$ & $\mathrm{~b}$ \\
10 & $\mathrm{~b}$ & $\mathrm{~b}$ & $\mathrm{~b}$ & $\mathrm{~b}$ & $\mathrm{c}$ \\
\hline
\end{tabular}

Note-(a) The subject heard opposed eight-note scale patterns; (b) the subject heard opposed tetrachord arch patterns; (c) the subject heard a pair of disjunct pitch streams.

Both Tables 2 and 3 indicate a curious response pattern to Examples 4 and 5: in each test group, more subjects demonstrated the expected channeling behavior in response to Example 5 (where the intensities of all ascending partials from $800 \mathrm{~Hz}$ were increased $10 \mathrm{~dB}$ ) than in response to Example 4 (where the intensities of all ascending partials from $800 \mathrm{~Hz}$ were increased only $5 \mathrm{~dB}$ ).

It is also interesting that, when subjects were asked to describe any difference in Examples 4 and 5 as compared to Example 3, all subjects noted a definite change in tone quality (the most commonly used adjectives were "thinner" and "tinnier"), but no subject noted that the altered tone quality was confined to only one pitch series, or that it emanated from only one headphone or speaker. Instead, the subjects stated uniformly that they had heard these timbral changes in both pitch sequences, and in both ears.

\section{Experiment 2}

The stimuli were presented dichotically to 10 subjects, and binaurally to 12 subjects. Table 1 shows the responses of these subject groups in percentages. With one exception, all subjects perceived these pitch sequences as pairs of tetrachordal arches in contrary motion. The exceptional response occurred, as did those in Experiment 1, in the binaural subject group: one subject notated Example 2 (sine tones) as a pair of eight-note scale patterns in contrary motion.

\section{Experiment 3}

The stimuli were presented dichotically to 13 subjects, and binaurally to 15 subjects. All notated responses were virtually identical to the contrapuntal patterns shown in Figures 2a and 2c.

If subjects noted, at any point in the test, that melodic lines appeared to switch from ear to ear, they were instructed to mark each instance of switching on their answer sheets. Additionally, all answer sheets were examined to determine whether the pitch interval of any notational error resembled, in its melodic direction, the corresponding interval in the patterns shown in Figures $2 \mathrm{~b}$ or $2 \mathrm{~d}$. Any such resemblance, no matter how tenuous, was considered to be an instance of ear-to-ear melodic switching (i.e., a breakdown in the channeling response). There were 12 such instances of ear-to-ear switching among the dichotic test group: one subject perceived 8 of these switches, one subject perceived 3, and one subject perceived 1. Significantly, no two subjects heard any of these switches at the same point in either example. As a group, the 13 subjects in the dichotic group notated a combined total of 572 melodic intervals, and heard 12 of those intervals $(2 \%)$ switch from ear to ear. The responses of one subject accounted for two-thirds of this percentage. After one test session, one subject decided that Example 2 contained ear-to-ear switches on every note; this was the only such observation made by any subject employed in this study. The observation was considered to be anomalous, since the subject's notated response exactly resembled Figure 2c.

There were six instances of ear-to-ear switching among the binaural test group: one subject perceived two of these switches, and four subjects perceived one switch each. In contrast to the response pattern within the dichotic test group, four of the five subjects in the binaural group who perceived an ear-toear switch agreed on its placement: between Notes 5 and 6 of Example 1. There was no agreement among these subjects as to the temporal placement of any other ear-to-ear switch. As a group, the 15 subjects in the binaural test group notated a combined total of 660 melodic intervals, and heard 6 of those intervals $(.9 \%)$ switch from ear to ear.

No subject heard any additional ear-to-ear switches when the examples were played again with unequal frequency responses at the two headphones or speakers. Responses at this point were quite similar to those in Experiment 1: all subjects noted a change in tone quality, and all subjects stated that this change occurred in both melodic lines and in both headphone or speaker signals.

\section{CONCLUSIONS}

It was found that the perceptual behavior of channeling by pitch is largely unaffected when several other stimulus parameters are changed. Melodic channeling behavior was exhibited in $95.2 \%$ of the responses to the scalar stimuli in Experiments 1 and 2 and in $99.1 \%$ of the responses to the melodic stimuli in Experiment 3.

Channeling occurs in a binaural (stereophonic free 
sound field) environment, as well as in a dichotic one. The only anomalous responses resulting from binaural presentation of the stimuli occurred in Experiments 1 and 2: a small percentage (4.8\%) of responses indicated that an eight-note scale pattern in contrary motion (Figure 3a) was heard, instead of the more commonly heard opposed tetrachordal arch patterns (Figure $3 \mathrm{~b}$ ). The perception of such eight-note scale patterns lies at variance with earlier findings (Deutsch, 1975); it is plausible that this inconsistency is attributable to either (a) a perceptual alteration resulting from free sound field presentation, or (b) dissimilarities in the subject populations used in the two studies. All subjects in this study were musicians with at least 1 year of training, which includes the writing and auditing of a great number of eight-note scale patterns. This training includes little, if any, such experience with patterns such as that shown in Figure 3b.

It appears that channeling by pitch range is so strong as to persist through a broad range of timbral changes and changes in envelope characteristics of stimulus tones, imperfectly timed attacks, durational inconsistencies (both articulative and metric), and varying loudnesses. One of the more remarkable observations was that no subject was able to correctly define dissimilar spectral contours when these switched from ear to ear. Instead, every subject reported an apparent change of tone quality which resided in both scalar or melodic sequences, and which apparently emanated from both headphones or speakers.

The melodic channeling percept seems to be largely unrelated to familiarity of subjects with melodic patterns used as stimuli. Although the stimuli used in Experiment 3 were simple and highly tonal, subjects were not nearly as familiar with the individual characteristics of those particular musical specimens as they were with $\mathrm{C}$ major scale patterns. Since subjects in this study were permitted to listen to the stimuli as many times as they wanted, and were not asked to make ex post facto descriptive reports on the stimuli or to "shadow sing" along with them, it is apparent that memory and vocal limitations of subjects play a negligible role in melodic channeling behavior.

\section{REFERENCES}

Deursch, D. An auditory illusion. Nature, 1974, 251, 307-309.

DEuTsCH, D. Two-channel listening to musical scales. Journal of the Acoustical Society of America, 1975, 57, 1156-1160.

Deutsch, D., \& Roll, P. Separate 'what' and 'where' decision mechanisms in processing a dichotic tonal sequence. Journal of Experimental Psychology: Human Perception and Performance, 1976, 2, 23-29.

DEUTSCH, D. Lateralization by frequency for repeating sequences of dichotic 400- and $800-\mathrm{Hz}$ tones. Journal of the Acoustical Society of America, 1978, 63, 184-186.

(Received for publication October 17, 1978; revision accepted January 2, 1979.) 